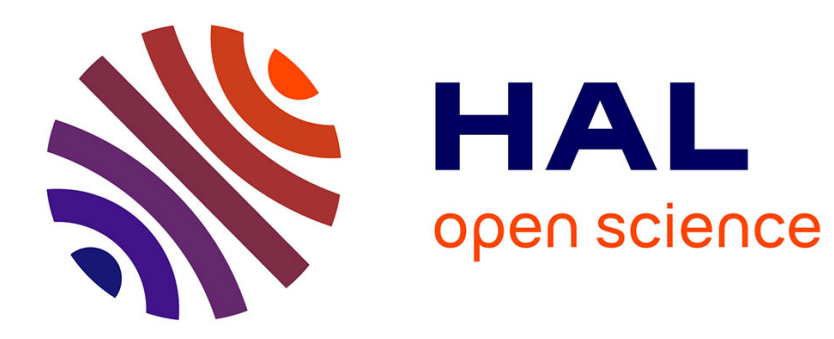

\title{
Evaluations of the Hankel determinants of a Thue-Morse-like sequence
}

\author{
Guo-Niu Han, Wen Wu
}

\section{To cite this version:}

Guo-Niu Han, Wen Wu. Evaluations of the Hankel determinants of a Thue-Morse-like sequence. International Journal of Number Theory, 2015, 11 (6), 10.1142/S1793042115500815 . hal-01278054

\section{HAL Id: hal-01278054 \\ https://hal.science/hal-01278054}

Submitted on 25 Feb 2016

HAL is a multi-disciplinary open access archive for the deposit and dissemination of scientific research documents, whether they are published or not. The documents may come from teaching and research institutions in France or abroad, or from public or private research centers.
L'archive ouverte pluridisciplinaire HAL, est destinée au dépôt et à la diffusion de documents scientifiques de niveau recherche, publiés ou non, émanant des établissements d'enseignement et de recherche français ou étrangers, des laboratoires publics ou privés. 


\title{
EVALUATIONS OF THE HANKEL DETERMINANTS OF A THUE-MORSE-LIKE SEQUENCE
}

\author{
GUO-NIU HAN AND WEN WU*
}

\begin{abstract}
We obtain simple relations between the Hankel determinants of the formal power series $\prod_{k>0}\left(1+J x^{3^{k}}\right)$ where $J=$ $(\sqrt{-3}-1) / 2$, and prove that the sequence of Hankel determinants is an aperiodic automatic sequence taking value in $\left\{ \pm 1, \pm J, \pm J^{2}\right\}$. This research is essentially inspired by the works about Hankel determinants of Thue-Morse-like sequences by Allouche, Peyrière, Wen and Wen (1998), Bacher (2006) and the first author (2013). It is worth mentioning that we do not have a unified result, even though Bacher's result and ours are very similar.
\end{abstract}

\section{INTRODUCTION}

In 1998, Allouche, Peyrière, Wen and Wen proved that all the Hankel determinants of the Thue-Morse sequence defined by the generating function

$$
P_{2}(x)=\prod_{k \geq 0}\left(1-x^{2^{k}}\right)
$$

are non-zero by using determinant manipulation [1], which consists of proving sixteen recurrent relations between determinants. Recently, the first author derived a short proof of APWW's result by using the Jacobi continued fraction expansion of the underlying sequence [9]. Moreover, he proved that all the Hankel determinants of the ThueMorse-like sequence defined by the generating function

$$
P_{3}(x)=\prod_{k \geq 0}\left(1-x^{3^{k}}\right)
$$

are non-zero. These results about Hankel determinants have been shown to have useful applications in Number Theory for studying the irrationality exponents of some automatic numbers (see $[7,8]$ ).

Date: September 19, 2014.

2010 Mathematics Subject Classification. 05A15, 11B37, 11B85, 11C20, 15A15.

Key words and phrases. Hankel determinant, Thue-Morse sequence, ThueMorse-like sequence, automatic sequence.

This research is supported by NSFC (Grant Nos. 11401188, 11371156).

* Wen $\mathrm{Wu}$ is the corresponding author. 
Notice that the Hankel determinants of $P_{2}(x)$ and $P_{3}(x)$ do not seem to have any closed-form expressions. The trick to study such determinants is using the modular arithmetic. Surprisingly enough, Bacher obtained simple relations between the Hankel determinants of the ThueMorse-like sequence defined by the generating function

$$
P_{2}(x ; I)=\prod_{k \geq 0}\left(1+I x^{2^{k}}\right),
$$

where $I$ is the imaginary unit $[3,4]$. Bacher's method is based on the category Rec introduced by himself. Inspired by the above three results, we derive simple relations between the Hankel determinants of the Thue-Morse-like sequence defined by the generating function

$$
P_{3}(x ; J)=\prod_{k \geq 0}\left(1+J x^{3^{k}}\right),
$$

where $J=(I \sqrt{3}-1) / 2$. Our proof is based on APWW's method by using direct determinant manipulations.

Consider the sequence $\boldsymbol{c}=\left(c_{0}, c_{1}, c_{2}, \cdots\right)$ defined by the generating function

$$
\begin{gathered}
P_{3}(x ; J)=\prod_{k \geq 0}\left(1+J x^{3^{k}}\right)=c_{0}+c_{1} x+c_{2} x^{2}+\cdots \\
=1+J x+J x^{3}+J^{2} x^{4}+J x^{9}+J^{2} x^{10}+J^{2} x^{12}+x^{13}+J x^{27}+\cdots
\end{gathered}
$$

It is easy to show that the sequence $c$ can be characterized by the following recurrence relations

$$
c_{0}=1, \quad c_{3 n}=c_{n}, \quad c_{3 n+1}=J c_{n}, \quad c_{3 n+2}=0, \quad(n \geq 0)
$$

or equivalently by the morphism $\sigma$ over alphabet $\mathcal{A}=\left\{0,1, J, J^{2}\right\}$ where $\sigma$ is defined as follow

$$
1 \mapsto 1 J 0, \quad J \mapsto J J^{2} 0, \quad J^{2} \mapsto J^{2} 10, \quad 0 \mapsto 000 .
$$

Thus $\boldsymbol{c}$ is an automatic sequence, i.e., it can be generated by a finite automaton (see [2]).

Recall that for each sequence of complex numbers $\mathbf{u}=\left(u_{k}\right)_{k=0,1, \ldots}$ the corresponding $(p, n)$-order Hankel matrix $H_{n}^{p}(\mathbf{u})$ is given by

$$
H_{n}^{p}(\mathbf{u})=\left(\begin{array}{cccc}
u_{p} & u_{p+1} & \cdots & u_{p+n-1} \\
u_{p+1} & u_{p+2} & \cdots & u_{p+n} \\
\cdots & \cdots & \cdots & \cdots \\
u_{p+n-1} & u_{p+n} & \cdots & u_{p+2 n-2}
\end{array}\right),
$$

where $n \geq 1$ and $p \geq 0$. The Hankel determinant $\left|H_{n}^{p}(\mathbf{u})\right|$ is simply the determinant of the Hankel matrix $H_{n}^{p}(\mathbf{u})$. By convention, $\left|H_{0}^{p}(\mathbf{u})\right|=1$. 
Our main result about the Hankel determinants of $\boldsymbol{c}$ is stated next.

Theorem 1. Let $H_{n}^{p}:=H_{n}^{p}(\boldsymbol{c})$ be the $(p, n)$-order Hankel matrix of the Thue-Morse-like sequence $\boldsymbol{c}$ defined in (5). Then, the Hankel determinants $\left|H_{n}^{0}\right|$ and $\left|H_{n}^{1}\right|$ are characterized by the following recurrence relations

$$
\left\{\begin{aligned}
\left|H_{0}^{0}\right| & =1 \\
\left|H_{1}^{0}\right| & =1 \\
\left|H_{3 n}^{0}\right| & =\left|H_{n}^{0}\right|, \\
\left|H_{3 n+1}^{0}\right| & =\left|H_{n+1}^{0}\right| \\
\left|H_{3 n+2}^{0}\right| & =-J^{2}\left|H_{n+1}^{0}\right|
\end{aligned}\right.
$$

and

for all $n \geq 0$.

$$
\left\{\begin{aligned}
\left|H_{0}^{1}\right| & =1 \\
\left|H_{3 n}^{1}\right| & =\left|H_{n}^{1}\right|, \\
\left|H_{3 n+1}^{1}\right| & =J\left|H_{n}^{1}\right| \\
\left|H_{3 n+2}^{1}\right| & =J\left|H_{n+1}^{1}\right|
\end{aligned}\right.
$$

The first values of the Hankel determinants $\left|H_{n}^{0}\right|$ and $\left|H_{n}^{1}\right|$ are reproduced in the following table.

$$
\begin{array}{cccccccccccccc}
n & = & 0 & 1 & 2 & 3 & 4 & 5 & 6 & 7 & 8 & 9 & 10 & 11 \\
\left|H_{n}^{0}\right| & = & 1 & 1 & -J^{2} & 1 & -J^{2} & J & -J^{2} & 1 & -J^{2} & 1 & -J^{2} & J \\
\left|H_{n}^{1}\right| & = & 1 & J & J^{2} & J & J^{2} & 1 & J^{2} & 1 & J^{2} & J & J^{2} & 1
\end{array}
$$

Consider the sequence $\boldsymbol{s}=\left(s_{0}, s_{1}, s_{2}, \cdots\right)$ defined by $s_{n}=c_{n}+c_{n+1}$. We have

$$
\begin{gathered}
S(x)=\frac{(1+x) P_{3}(x ; J)-1}{x}=s_{0}+s_{1} x+s_{2} x^{2}+\cdots \\
=-J^{2}+J x+J x^{2}-x^{3}+J^{2} x^{4}+J x^{8}-x^{9}+J^{2} x^{10}+\cdots
\end{gathered}
$$

and

$$
s_{3 n}=-J^{2} c_{n}, s_{3 n+1}=J c_{n}, s_{3 n+2}=c_{n+1} .
$$

The Hankel matrices of the sequences $\mathbf{s}$ are denoted by $\Sigma_{n}^{p}$. An easy observation shows that

$$
\Sigma_{n}^{p}=H_{n}^{p}+H_{n}^{p+1}
$$

Theorem 2. The Hankel determinants $\left|\Sigma_{n}^{0}\right|$ and $\left|\Sigma_{n}^{1}\right|$ are characterized by the following recurrent relations

$$
\left\{\begin{aligned}
\left|\Sigma_{0}^{0}\right| & =1 \\
\left|\Sigma_{3 n}^{0}\right| & =\left|\Sigma_{n}^{0}\right| \\
\left|\Sigma_{3 n+1}^{0}\right| & =-J^{2}\left|\Sigma_{n}^{0}\right| \\
\left|\Sigma_{3 n+2}^{0}\right| & =-J^{2}\left|\Sigma_{n+1}^{0}\right|
\end{aligned}\right.
$$


and

$$
\left\{\begin{aligned}
\left|\Sigma_{0}^{1}\right| & =1 \\
\left|\Sigma_{3 n}^{1}\right| & =\left|\Sigma_{n}^{1}\right| \\
\left|\Sigma_{3 n+1}^{1}\right| & =J\left|\Sigma_{n}^{1}\right| \\
\left|\Sigma_{3 n+2}^{1}\right| & =\left|\Sigma_{n}^{1}\right|
\end{aligned}\right.
$$

for all $n \geq 0$.

The first values of the Hankel determinants $\left|\Sigma_{n}^{0}\right|$ and $\left|\Sigma_{n}^{1}\right|$ are reproduced in the following table.

$$
\begin{array}{cccccccccccccc}
n & = & 0 & 1 & 2 & 3 & 4 & 5 & 6 & 7 & 8 & 9 & 10 & 11 \\
\left|\Sigma_{n}^{0}\right| & = & 1 & -J^{2} & J & -J^{2} & J & -1 & J & -1 & J & -J^{2} & J & -1 \\
\left|\Sigma_{n}^{1}\right| & = & 1 & J & 1 & J & J^{2} & J & 1 & J & 1 & J & J^{2} & J
\end{array}
$$

Theorem 3. The four sequences of Hankel determinants $\left(\left|H_{n}^{0}\right|\right)_{n \geq 0}$, $\left(\left|H_{n}^{1}\right|\right)_{n \geq 0}, \quad\left(\left|\Sigma_{n}^{0}\right|\right)_{n \geq 0}$ and $\left(\left|\Sigma_{n}^{1}\right|\right)_{n \geq 0}$ are all aperiodic taking value in $\left\{ \pm 1, \pm J, \pm J^{2}\right\}$.

Let us make further comments about Hankel determinants and automatic sequences. Hankel matrices and Hankel determinants of a sequence are strongly connected to the moment problem [12] and to the Padé approximations $[5,6]$. Allouche, Peyrière, Wen and Wen studied the properties of Hankel determinants $\left|\mathcal{E}_{n}^{p}\right|$ of the Thue-Morse sequence in [1]. They proved that the two-dimensional sequence (or double sequence) of the Hankel determinants $\left|\mathcal{E}_{n}^{p}\right|$ modulo 2 is 2-automatic. In [11], Kamae, Tamura and Wen studied the properties of Hankel determinants for the Fibonacci word and gave a quantitative relation between the Hankel determinant and the Padé pair. Later, Tamura [13] generalized the results for a class of special sequences.

Theorem 1 implies that the first two columns of the two-dimensional sequence $\left\{\left|H_{n}^{p}\right|\right\}_{n, p \geq 0}$, i.e., $\left\{\left|H_{n}^{0}\right|\right\}_{n \geq 0}$ and $\left\{H_{n}^{1}\right\}_{n \geq 0}$, are 3 -automatic sequences. They are aperiodic, this is different from the Hankel determinants of the Thue-Morse sequence and from the regular paperfolding sequence studied in $[1]$ and $[8,10]$ respectively.

In Section 2 we establish a key lemma, namely, Lemma 5, which consists of a list of recurrence relations between the determinants $\left|H_{n}^{p}\right|$ and $\left|\Sigma_{n}^{p}\right|$. Theorems 1-3 are simple consequences of Lemma 5. The recurrence relations them-self are proved in Sections 3 and 4 .

\section{The Sudoku Method}

The proofs of Theorems 1-3 are based on the method developed by Allouche, Peyrière, Wen and Wen [1], that could be called sudoku 
method. The sudoku method consists of some basic determinant manipulations. Matrices are often split into $3 \times 3=9$ small blocks.

For each matrix $M=\left(m_{i, j}\right)_{i, j=1,2, \ldots, n}$ of size $n \times n$, denote by $M^{t}$ the transpose of $M$. Let $M^{(i)}$ be the $n \times(n-1)$-matrix obtained by deleting the $i$-th column of $M$, and $M_{(i)}$ be the $(n-1) \times n$-matrix obtained by deleting the $i$-th row of $M$. The determinant of the matrix $M$ is denoted by $|M|$. Also, let $\mathbf{0}_{m, n}$ denote the $m \times n$ zero matrix and $\mathbf{I}_{n}$ denote the identity matrix of order $n$.

For each $n \geq 1$ let $P(n)$ be the $n \times n$-matrix defined by

$$
P(n)=\left(e_{1}, e_{4}, \cdots, e_{3 n_{1}-2}, e_{2}, e_{5}, \cdots, e_{3 n_{2}-1}, e_{3}, e_{6}, \cdots, e_{3 n_{3}}\right),
$$

where $n_{1}=\left\lfloor\frac{n+2}{3}\right\rfloor, n_{2}=\left\lfloor\frac{n+1}{3}\right\rfloor, n_{3}=\left\lfloor\frac{n}{3}\right\rfloor$ and $e_{j}$ is the $j$-th unit column vector of order $n$, i.e., the column vector with 1 as the $j$-th entry and zeros elsewhere. For simplicity, we write $P$ instead of $P(n)$, when no confusion can occur. Obviously, $|P(n)|= \pm 1$. When consider $P(3 n), P(3 n+1), P(3 n+2)$, the following diagram shows the values of $n_{1}, n_{2}$ and $n_{3}$ in these cases:

\begin{tabular}{c|ccc} 
& $n_{1}$ & $n_{2}$ & $n_{3}$ \\
\hline $3 n$ & $n$ & $n$ & $n$ \\
$3 n+1$ & $n+1$ & $n$ & $n$ \\
$3 n+2$ & $n+1$ & $n+1$ & $n$
\end{tabular}

The proof of the following lemma is left to the reader.

Lemma 4. Let $M=\left(m_{i, j}\right)_{1 \leq i, j \leq n}$ be an $n \times n$-matrix and $P=P(n)$ be the matrix, of the same size as $M$, defined in (15). Then

$$
P^{t} M P=\left(\begin{array}{ccc}
\left(m_{3 i-2,3 j-2}\right)_{n_{1} \times n_{1}} & \left(m_{3 i-2,3 j-1}\right)_{n_{1} \times n_{2}} & \left(m_{3 i-2,3 j}\right)_{n_{1} \times n_{3}} \\
\left(m_{3 i-1,3 j-2}\right)_{n_{2} \times n_{1}} & \left(m_{3 i-1,3 j-1}\right)_{n_{2} \times n_{2}} & \left(m_{3 i-1,3 j}\right)_{n_{2} \times n_{3}} \\
\left(m_{3 i, 3 j-2}\right)_{n_{3} \times n_{1}} & \left(m_{3 i, 3 j-1}\right)_{n_{3} \times n_{2}} & \left(m_{3 i, 3 j}\right)_{n_{3} \times n_{3}}
\end{array}\right),
$$

where $\left(m_{3 i-2,3 j-1}\right)_{s \times t}$ means the matrix $\left(m_{3 i-2,3 j-1}\right)_{1 \leq i \leq s, 1 \leq j \leq t}$.

Recall that for each sequence of complex numbers $\mathbf{u}=\left(u_{k}\right)_{k=0,1, \ldots}$ the corresponding $(p, n)$-order Hankel matrix $H_{n}^{p}(\mathbf{u})$ is defined by $(7)$. Let $K_{n}^{p}=K_{n}^{p}(\mathbf{u}):=\left(u_{p+3(i+j-2)}\right)_{1 \leq i, j \leq n}$. When applying Lemma 4 for $M=H_{3 n}^{p}(\mathbf{u}), H_{3 n+1}^{p}(\mathbf{u}), H_{3 n+2}^{p}(\mathbf{u})$, we get

$$
\begin{aligned}
& P^{t} H_{3 n}^{p}(\mathbf{u}) P \\
= & \left(\begin{array}{ccc}
\left(u_{p+3(i+j-2)}\right)_{n \times n} & \left(u_{p+3(i+j-2)+1}\right)_{n \times n} & \left(u_{p+3(i+j-2)+2}\right)_{n \times n} \\
\left(u_{p+3(i+j-2)+1}\right)_{n \times n} & \left(u_{p+3(i+j-2)+2}\right)_{n \times n} & \left(u_{p+3(i+j-2)+3}\right)_{n \times n} \\
\left(u_{p+3(i+j-2)+2}\right)_{n \times n} & \left(u_{p+3(i+j-2)+3}\right)_{n \times n} & \left(u_{p+3(i+j-2)+4}\right)_{n \times n}
\end{array}\right)
\end{aligned}
$$




$$
=\left(\begin{array}{ccc}
K_{n}^{p} & K_{n}^{p+1} & K_{n}^{p+2} \\
K_{n}^{p+1} & K_{n}^{p+2} & K_{n}^{p+3} \\
K_{n}^{p+2} & K_{n}^{p+3} & K_{n}^{p+4}
\end{array}\right),
$$

and

$$
\begin{aligned}
P^{t} H_{3 n+1}^{p}(\mathbf{u}) P & =\left(\begin{array}{ccc}
K_{n+1}^{p} & \left(K_{n+1}^{p+1}\right)^{(n+1)} & \left(K_{n+1}^{p+2}\right)^{(n+1)} \\
\left(K_{n+1}^{p+1}\right)_{(n+1)} & K_{n}^{p+2} & K_{n}^{p+3} \\
\left(K_{n+1}^{p+2}\right)_{(n+1)} & K_{n}^{p+3} & K_{n}^{p+4}
\end{array}\right), \\
P^{t} H_{3 n+2}^{p}(\mathbf{u}) P & =\left(\begin{array}{ccc}
K_{n+1}^{p} & K_{n+1}^{p+1} & \left(K_{n+1}^{p+2}\right)^{(n+1)} \\
K_{n+1}^{p+1} & K_{n+1}^{p+2} & \left(K_{n+1}^{p+3}\right)^{(n+1)} \\
\left(K_{n+1}^{p+2}\right)_{(n+1)} & \left(K_{n+1}^{p+3}\right)_{(n+1)} & K_{n}^{p+4}
\end{array}\right) .
\end{aligned}
$$

As shown in Sections 3-4, Formulae (16)-(18) can be used to prove the following recurrence relations between the determinants $\left|H_{n}^{p}\right|$ and $\left|\Sigma_{n}^{p}\right|$ when the sequence $\mathbf{u}$ is taken from the sequences $\mathbf{c}$ and $\mathbf{s}$ defined in (6) and (11) respectively. Through these eighteen recurrence formulae, we can evaluate all the Hankel determinants $\left|H_{n}^{p}\right|$ and $\left|\Sigma_{n}^{p}\right|(n \geq 1, p \geq 0)$. Our key lemma is stated next.

Lemma 5. For each $p \geq 0$ and $n \geq 1$ we have

$$
\begin{aligned}
& \text { (L1) }\left|H_{3 n}^{3 p}\right|=(-1)^{n}\left|H_{n}^{p}\right| \cdot\left|H_{n}^{p+1}\right| \cdot\left|\Sigma_{n}^{p}\right| \text {, } \\
& \text { (L2) }\left|H_{3 n+1}^{3 p}\right|=(-1)^{n}\left|H_{n}^{p+1}\right| \cdot\left|H_{n+1}^{p}\right| \cdot\left|\Sigma_{n}^{p}\right| \text {, } \\
& \text { (L3) }\left|H_{3 n+2}^{3 p}\right|=(-1)^{n+1} J^{2}\left|H_{n+1}^{p}\right|^{2} \cdot\left|\Sigma_{n}^{p+1}\right| \text {, } \\
& \text { (L4) }\left|H_{3 n}^{3 p+1}\right|=(-1)^{n}\left|H_{n}^{p+1}\right|^{2} \cdot\left|\Sigma_{n}^{p}\right| \text {, } \\
& \text { (L5) }\left|H_{3 n+1}^{3 p+1}\right|=(-1)^{n} J\left|H_{n}^{p+1}\right| \cdot\left|H_{n+1}^{p}\right| \cdot\left|\Sigma_{n}^{p+1}\right| \text {, } \\
& \text { (L6) }\left|H_{3 n+2}^{3 p+1}\right|=(-1)^{n} J\left|H_{n+1}^{p+1}\right| \cdot\left|H_{n+1}^{p}\right| \cdot\left|\Sigma_{n}^{p+1}\right| \text {, } \\
& \text { (L7) }\left|H_{3 n}^{3 p+2}\right|=(-1)^{n}\left|H_{n}^{p+1}\right|^{2} \cdot\left|\Sigma_{n}^{p+1}\right| \text {, } \\
& \text { (L8) }\left|H_{3 n+1}^{3 p+2}\right|=0 \text {, } \\
& \text { (L9) }\left|H_{3 n+2}^{3 p+2}\right|=(-1)^{n+1}\left|H_{n+1}^{p+1}\right|^{2} \cdot\left|\Sigma_{n}^{p+1}\right| \text {, } \\
& \text { (L10) }\left|\Sigma_{3 n}^{3 p}\right|=(-1)^{n}\left|\Sigma_{n}^{p}\right|^{2} \cdot\left|H_{n}^{p+1}\right| \text {, } \\
& \text { (L11) }\left|\Sigma_{3 n+1}^{3 p}\right|=(-1)^{n+1} J^{2}\left|H_{n+1}^{p}\right| \cdot\left|\Sigma_{n}^{p}\right| \cdot\left|\Sigma_{n}^{p+1}\right| \text {, } \\
& \text { (L12) }\left|\Sigma_{3 n+2}^{3 p}\right|=(-1)^{n+1} J^{2}\left|H_{n+1}^{p}\right| \cdot\left|\Sigma_{n+1}^{p}\right| \cdot\left|\Sigma_{n}^{p+1}\right| \text {, } \\
& \text { (L13) }\left|\Sigma_{3 n}^{3 p+1}\right|=(-1)^{n}\left|H_{n}^{p+1}\right| \cdot\left|\Sigma_{n}^{p}\right| \cdot\left|\Sigma_{n}^{p+1}\right| \text {, } \\
& \text { (L14) }\left|\Sigma_{3 n+1}^{3 p+1}\right|=(-1)^{n} J\left|H_{n+1}^{p}\right| \cdot\left|\Sigma_{n}^{p+1}\right|^{2} \text {, } \\
& \text { (L15) }\left|\Sigma_{3 n+2}^{3 p+1}\right|=(-1)^{n+1}\left|H_{n+1}^{p+1}\right| \cdot\left|\Sigma_{n+1}^{p}\right| \cdot\left|\Sigma_{n}^{p+1}\right|, \\
& \text { (L16) }\left|\Sigma_{3 n}^{3 p+2}\right|=(-1)^{n}\left|\Sigma_{n}^{p+1}\right|^{2} \cdot\left|H_{n}^{p+1}\right| \text {, } \\
& \text { (L17) }\left|\Sigma_{3 n+1}^{3 p+2}\right|=(-1)^{n}\left|\Sigma_{n}^{p+1}\right|^{2} \cdot\left|H_{n+1}^{p+1}\right| \text {, } \\
& \text { (L18) }\left|\Sigma_{3 n+2}^{3 p+2}\right|=0 \text {. }
\end{aligned}
$$


Corollary 6. Let $A_{n}^{p}=(-1)^{n}\left|H_{n}^{p+1}\right| \cdot\left|\Sigma_{n}^{p}\right|$ and $B_{n}^{p}=(-1)^{n}\left|H_{n+1}^{p}\right|$. $\left|\Sigma_{n}^{p+1}\right|$. Then,

(C1) $A_{3 n}^{3 p}=\left(A_{n}^{p}\right)^{3}$,

(C2) $A_{3 n+1}^{3 p}=A_{n}^{p}\left(B_{n}^{p}\right)^{2}$

(C3) $A_{3 n+2}^{3 p}=A_{n+1}^{p}\left(B_{n}^{p}\right)^{2}$,

(C4) $B_{3 n}^{3 p}=\left(A_{n}^{p}\right)^{2} B_{n}^{p}$,

(C5) $B_{3 n+1}^{3 p}=\left(B_{n}^{p}\right)^{3}$,

(C6) $B_{3 n+2}^{3 p}=\left(A_{n+1}^{p}\right)^{2} B_{n}^{p}$.

Proof. (C1) From Equalities (L4) and (L10) stated in Lemma 5 we have

$$
\begin{aligned}
A_{3 n}^{3 p} & =(-1)^{3 n}\left|H_{3 n}^{3 p+1}\right| \cdot\left|\Sigma_{3 n}^{3 p}\right| \\
& =(-1)^{3 n}(-1)^{n}\left|H_{n}^{p+1}\right|^{2}\left|\Sigma_{n}^{p}\right| \cdot(-1)^{n}\left|\Sigma_{n}^{p}\right|^{2}\left|H_{n}^{p+1}\right| \\
& =(-1)^{3 n}\left|H_{n}^{p+1}\right|^{3}\left|\Sigma_{n}^{p}\right|^{3} \\
& =\left(A_{n}^{p}\right)^{3} .
\end{aligned}
$$

Identity $(C 2)$ (resp. $(C 3),(C 4),(C 5),(C 6))$ are proved in the same manner by using Equalities (L5) and (L11) (resp. (L6) and (L12), (L2) and $(L 13),(L 3)$ and $(L 14),(L 1)$ and $(L 15))$ stated in Lemma 5.

Proof of Theorems 1-2. Let $p_{n}=(-1)^{n}\left|H_{n}^{1}\right| \cdot\left|\Sigma_{n}^{0}\right|$ and $q_{n}=(-1)^{n}\left|H_{n+1}^{0}\right|$. $\left|\Sigma_{n}^{1}\right|$. Then Corollary 6 shows that

(1) $p_{3 n}=p_{n}^{3}$,

(2) $p_{3 n+1}=p_{n} q_{n}^{2}$,

(3) $p_{3 n+2}=p_{n+1} q_{n}^{2}$

(4) $q_{3 n}=p_{n}^{2} q_{n}$,

(5) $q_{3 n+1}=q_{n}^{3}$,

(6) $q_{3 n+2}=p_{n+1}^{2} q_{n}$.

Moreover, the first values are $p_{0}=p_{1}=p_{2}=q_{0}=q_{1}=q_{2}=1$. By induction, we have $p_{n}=q_{n}=1$ for all $n \geq 0$. In other words,

$$
\left|H_{n}^{1}\right| \cdot\left|\Sigma_{n}^{0}\right|=(-1)^{n} \quad \text { and } \quad\left|H_{n+1}^{0}\right| \cdot\left|\Sigma_{n}^{1}\right|=(-1)^{n} .
$$

The last three identities in (8) are consequences of Equations (L1), (L2), (L3) respectively. Similarly, the last three identities in (9) are consequences of Equations (L4), (L5), (L6) respectively. Thus, Theorem 1 is proved. By Relation (19), Theorem 1 implies Theorem 2.

Proof of Theorem 3. Suppose that $\left(\left|H_{n}^{0}\right|\right)_{n \geq 0}$ were periodic and $T>0$ were the smallest period. Thus, there exists a positive integer $N$ such 
that $\left|H_{k}^{0}\right|=\left|H_{k+T}^{0}\right|$ for all $k>N$. If $T=3 \tau(\operatorname{resp} . T=3 \tau+1, T=$ $3 \tau+2)$, then

$$
\begin{gathered}
\left|H_{k}^{0}\right| \stackrel{\text { by (8) }}{=}\left|H_{3 k}^{0}\right| \stackrel{\text { periodicity }}{=}\left|H_{3 k+3 \tau}^{0}\right| \stackrel{\text { by (8) }}{=}\left|H_{k+\tau}^{0}\right| \\
\text { (resp. }\left|H_{k}^{0}\right| \stackrel{\text { by (8) }}{=}\left|H_{3 k}^{0}\right| \stackrel{\text { periodicity }}{=}\left|H_{3 k+3 \tau+1}^{0}\right| \stackrel{\text { by (8) }}{=}\left|H_{k+\tau+1}^{0}\right|, \\
\left.\left|H_{k+1}^{0}\right| \stackrel{\text { by (8) }}{=}\left|H_{3 k+1}^{0}\right| \stackrel{\text { periodicity }}{=}\left|H_{3 k+3 \tau+3}^{0}\right| \stackrel{\text { by (8) }}{=}\left|H_{k+\tau+1}^{0}\right|\right)
\end{gathered}
$$

for all $k>N$. This implies that $\tau$ (resp. $\tau+1, \tau$ ) is a period of $\left(\left|H_{n}^{0}\right|\right)_{n \geq 0}$, which contradicts the fact that $T$ were the smallest period. In the same manner, suppose that $\left(\left|H_{n}^{1}\right|\right)_{n \geq 0}$ were periodic and $T>0$ were the smallest period. Thus, there exists a positive integer $N$ such that $\left|H_{k}^{1}\right|=\left|H_{k+T}^{1}\right|$ for all $k>N$. If $T=3 \tau$ (resp. $T=3 \tau+1, T=$ $3 \tau+2$ ), then

$$
\left|H_{k}^{1}\right| \stackrel{\text { by }(9)}{=}\left|H_{3 k}^{1}\right| \stackrel{\text { periodicity }}{=}\left|H_{3 k+3 \tau}^{1}\right| \stackrel{\text { by }(9)}{=}\left|H_{k+\tau}^{1}\right|
$$

(resp. $\left|H_{k}^{1}\right| \stackrel{\text { by (9) }}{=} J^{-1}\left|H_{3 k+1}^{1}\right| \stackrel{\text { periodicity }}{=} J^{-1}\left|H_{3 k+3 \tau+2}^{1}\right| \stackrel{\text { by }(9)}{=}\left|H_{k+\tau+1}^{1}\right|$,

$$
\left.\left|H_{k+1}^{1}\right| \stackrel{\text { by (9) }}{=} J^{-1}\left|H_{3 k+2}^{1}\right| \stackrel{\text { periodicity }}{=} J^{-1}\left|H_{3 k+3 \tau+4}^{1}\right| \stackrel{\text { by (9) }}{=}\left|H_{k+\tau+1}^{1}\right|\right)
$$

for all $k>N$. This implies that $\tau$ (resp. $\tau+1, \tau$ ) is a period of $\left(\left|H_{n}^{1}\right|\right)_{n \geq 0}$, which contradicts the fact that $T$ were the smallest period.

The aperiodicities for the sequences $\left(\left|\Sigma_{n}^{0}\right|\right)_{n \geq 0}$ and $\left(\left|\Sigma_{n}^{1}\right|\right)_{n \geq 0}$ are proved in the same manner.

Since the set $\left\{ \pm 1, \pm J, \pm J^{2}\right\}$ is closed under multiplication, all elements in the four sequences take their values in the set $\left\{ \pm 1, \pm J, \pm J^{2}\right\}$ by Relations (8), (9), (13) and (14).

\section{Proof of Equalities $(L 1)-(L 9)$}

Recall that the Thue-Morse-like sequence $\mathbf{c}=c_{0} c_{1} \cdots c_{n} \cdots \in \mathcal{A}^{\infty}$ is characterized by the recurrent equations in (6), and that $H_{n}^{p}:=$ $H_{n}^{p}(\boldsymbol{c})$ is the $(p, n)$-order Hankel matrix of $\boldsymbol{c}$. Let $K_{n}^{p}:=K_{n}^{p}(\mathbf{c}):=$ $\left(c_{p+3(i+j-2)}\right)_{1 \leq i, j \leq n}$. By (6), we have for all $n \geq 1, p \geq 0$,

$$
K_{n}^{3 p}=H_{n}^{p}, \quad K_{n}^{3 p+1}=J H_{n}^{p}, \quad K_{n}^{3 p+2}=\mathbf{0}_{n, n} .
$$

Equalities (L1)-(L8) are proved by combining (20) and (16-18) where the sequence $\mathbf{u}$ is specialized to $\mathbf{c}$. For simplicity, during the proof, we will denote $n+1$ and $p+1$ by $\bar{n}$ and $\bar{p}$ respectively. Also, let $I_{n, n}$ be the identity matrix of size $n \times n$.

(L1) Combining (20) and (16), we have

$$
\left|H_{3 n}^{3 p}\right|=\left|P^{t} H_{3 n}^{3 p} P\right|
$$




$$
\begin{aligned}
& =\left|\begin{array}{ccc}
H_{n}^{p} & J H_{n}^{p} & \mathbf{0}_{n, n} \\
J H_{n}^{p} & \mathbf{0}_{n, n} & H_{n}^{\bar{p}} \\
\mathbf{0}_{n, n} & H_{n}^{\bar{p}} & J H_{n}^{\bar{p}}
\end{array}\right| \\
& \left.=\mid \begin{array}{ccc}
H_{n}^{p} & J H_{n}^{p} & \mathbf{0}_{n, n} \\
J H_{n}^{p} & \mathbf{0}_{n, n} & H_{n}^{\bar{p}} \\
\mathbf{0}_{n, n} & H_{n}^{\bar{p}} & J H_{n}^{\bar{p}}
\end{array}\right)\left(\begin{array}{ccc}
I_{n, n} & -J I_{n, n} & \mathbf{0}_{n, n} \\
\mathbf{0}_{n, n} & I_{n, n} & \mathbf{0}_{n, n} \\
\mathbf{0}_{n, n} & -J^{2} I_{n, n} & I_{n, n}
\end{array}\right) \mid \\
& =\left|\begin{array}{ccc}
H_{n}^{p} & \mathbf{0}_{n, n} & \mathbf{0}_{n, n} \\
J H_{n}^{p} & -J^{2} H_{n}^{p}-J^{2} H_{n}^{\bar{p}} & H_{n}^{\bar{p}} \\
\mathbf{0}_{n, n} & \mathbf{0}_{n, n} & J H_{n}^{\bar{p}}
\end{array}\right| \\
& =(-1)^{n}\left|H_{n}^{p}\right| \cdot\left|H_{n}^{p+1}\right| \cdot\left|\Sigma_{n}^{p}\right| .
\end{aligned}
$$

(L2) Combining (20) and (17), we have

$$
\begin{aligned}
& \left|H_{3 n+1}^{3 p}\right|=\left|P^{t} H_{3 n+1}^{3 p} P\right| \\
= & \left|\begin{array}{ccc}
H_{\bar{n}}^{p} & \left(J H_{\bar{n}}^{p}\right)^{(\bar{n})} & \mathbf{0}_{\bar{n}, n} \\
\left(J H_{\bar{n}}^{p}\right)_{(\bar{n})} & \mathbf{0}_{n, n} & H_{n}^{\bar{p}} \\
\mathbf{0}_{n, \bar{n}} & H_{n}^{\bar{p}} & J H_{n}^{\bar{p}}
\end{array}\right| \\
= & \left.\mid \begin{array}{ccc}
H_{\bar{n}}^{p} & \left(J H_{\bar{n}}^{p}\right)^{(\bar{n})} & \mathbf{0}_{\bar{n}, n} \\
\left(J H_{\bar{n}}^{p}\right)_{(\bar{n})} & \mathbf{0}_{n, n} & H_{n}^{\bar{p}} \\
\mathbf{0}_{n, \bar{n}} & H_{n}^{\bar{p}} & J H_{n}^{\bar{p}}
\end{array}\right)\left(\begin{array}{ccc}
I_{\bar{n}, \bar{n}} & -J I_{\bar{n}, n} & \mathbf{0}_{\bar{n}, n} \\
\mathbf{0}_{n, \bar{n}} & I_{n, n} & 0 \\
\mathbf{0}_{n, \bar{n}} & -J^{2} I_{n, n} & I_{n, n}
\end{array}\right) \mid \\
= & \left|\begin{array}{ccc}
H_{\bar{n}}^{p} & \mathbf{0}_{\bar{n}, n} & \mathbf{0}_{\bar{n}, n} \\
\left(J H_{\bar{n}}^{p}\right)_{(\bar{n})} & -J^{2}\left(H_{n}^{p}+H_{n}^{\bar{p}}\right) & H_{n}^{\bar{p}} \\
\mathbf{0}_{n, \bar{n}} & \mathbf{0}_{n, n} & J H_{n}^{\bar{p}}
\end{array}\right| \\
= & (-1)^{n}\left|H_{n}^{p+1}\right| \cdot\left|H_{n+1}^{p}\right| \cdot\left|\Sigma_{n}^{p}\right| .
\end{aligned}
$$

(L3) Combining (20) and (18), we have

$$
\begin{aligned}
& \left|H_{3 n+2}^{3 p}\right|=\left|P^{t} H_{3 n+2}^{3 p} P\right| \\
= & \left|\begin{array}{ccc}
H_{\bar{n}}^{p} & J H_{\bar{n}}^{p} & \mathbf{0}_{\bar{n}, n} \\
J H_{\bar{n}}^{p} & \mathbf{0}_{\bar{n}, \bar{n}} & \left(H_{\bar{n}}^{\bar{p}}\right)^{(\bar{n})} \\
\mathbf{0}_{n, \bar{n}} & \left(H_{\bar{n}}^{\bar{p}}\right)_{(\bar{n})} & J H_{n}^{\bar{p}}
\end{array}\right| \\
= & \left.\mid \begin{array}{ccc}
H_{\bar{n}}^{p} & J H_{\bar{n}}^{p} & \mathbf{0}_{\bar{n}, n}^{p} \\
J H_{\bar{n}}^{p} & \mathbf{0}_{\bar{n}, \bar{n}} & \left(H_{\bar{n}}^{\bar{p}}\right)^{(\bar{n})} \\
\mathbf{0}_{n, \bar{n}} & \left(H_{\bar{n}}^{\bar{p}}\right)_{(\bar{n})} & J H_{n}^{\bar{p}}
\end{array}\right)\left(\begin{array}{ccc}
I_{\bar{n}, \bar{n}} & -J I_{\bar{n}, \bar{n}} & -J^{2}\left(I_{\bar{n}, \bar{n})^{(1)}}\right. \\
\mathbf{0}_{\bar{n}, \bar{n}} & I_{\bar{n}, \bar{n}} & J\left(I_{\bar{n}, \bar{n}}\right)^{(1)} \\
\mathbf{0}_{n, \bar{n}} & \mathbf{0}_{n, \bar{n}} & I_{n, n}
\end{array}\right) \mid \\
= & \left|\begin{array}{ccc}
H_{\bar{n}}^{p} & \mathbf{0}_{\bar{n}, \bar{n}} & \mathbf{0}_{\bar{n}, n} \\
J H_{\bar{n}}^{p} & -J^{2} H_{\bar{n}}^{p} & \mathbf{0}_{\bar{n}, n} \\
\mathbf{0}_{n, \bar{n}} & \left(H_{\bar{n}}^{\bar{p}}\right)_{(\bar{n})} & J\left(H_{n}^{\bar{p}}+H_{n}^{\bar{p}+1}\right)
\end{array}\right| \\
= & (-1)^{n+1} J^{2}\left|H_{n+1}^{p}\right|^{2} \cdot\left|\Sigma_{n}^{p+1}\right| .
\end{aligned}
$$


(L4) Combining (20) and (16), we have

$$
\begin{aligned}
& \left|H_{3 n}^{3 p+1}\right|=\left|P^{t} H_{3 n}^{3 p+1} P\right| \\
= & \left|\begin{array}{ccc}
J H_{n}^{p} & \mathbf{0}_{n, n} & H_{n}^{\bar{p}} \\
\mathbf{0}_{n, n} & H_{n}^{\bar{p}} & J H_{n}^{\bar{p}} \\
H_{n}^{\bar{p}} & J H_{n}^{\bar{p}} & \mathbf{0}_{n, n}
\end{array}\right| \\
= & \left.\mid \begin{array}{ccc}
J H_{n}^{p} & \mathbf{0}_{n, n} & H_{n}^{\bar{p}} \\
\mathbf{0}_{n, n} & H_{n}^{\bar{p}} & J H_{n}^{\bar{p}} \\
H_{n}^{\bar{p}} & J H_{n}^{\bar{p}} & \mathbf{0}_{n, n}
\end{array}\right)\left(\begin{array}{ccc}
I_{n, n} & -J I_{n, n} & J^{2} I_{n, n} \\
\mathbf{0}_{n, n} & I_{n, n} & -J I_{n, n} \\
\mathbf{0}_{n, n} & \mathbf{0}_{n, n} & I_{n, n}
\end{array}\right) \mid \\
= & \left|\begin{array}{ccc}
J H_{n}^{p} & -J^{2} H_{n}^{p} & H_{n}^{p}+H_{n}^{\bar{p}} \\
\mathbf{0}_{n \times n} & H_{n}^{\bar{p}} & \mathbf{0}_{n, n} \\
H_{n}^{\bar{p}} & \mathbf{0}_{n, n} & \mathbf{0}_{n \times n}
\end{array}\right| \\
= & (-1)^{n}\left|H_{n}^{p+1}\right|^{2} \cdot\left|\Sigma_{n}^{p}\right| .
\end{aligned}
$$

(L5) Combining (20) and (17), we have

$$
\begin{aligned}
& \left|H_{3 n+1}^{3 p+1}\right|=\left|P^{t} H_{3 n+1}^{3 p+1} P\right| \\
= & \left|\begin{array}{ccc}
J H_{\bar{n}}^{p} & \mathbf{0}_{\bar{n}, n} & \left(H_{\bar{n}}^{\bar{p}}\right)^{(\bar{n})} \\
\mathbf{0}_{n, \bar{n}} & H_{n}^{\bar{p}} & J H_{n}^{\bar{p}} \\
\left(H_{\bar{n}}^{\bar{p}}\right)_{(\bar{n})} & J H_{n}^{\bar{p}} & \mathbf{0}_{n, n}
\end{array}\right| \\
= & \left.\mid \begin{array}{ccc}
J H_{\bar{n}}^{p} & \mathbf{0}_{\bar{n}, n} & \left(H_{\bar{n}}^{\bar{p}}\right)^{(\bar{n})} \\
\mathbf{0}_{n, \bar{n}} & H_{n}^{\bar{p}} & J H_{n}^{\bar{p}} \\
\left(H_{\bar{n}}^{\bar{p}}\right)_{(\bar{n})} & J H_{n}^{\bar{p}} & \mathbf{0}_{n, n}
\end{array}\right)\left(\begin{array}{ccc}
I_{n, n} & \mathbf{0}_{\bar{n}, n} & -J^{2}\left(I_{\bar{n}, \bar{n}}\right)^{(1)} \\
\mathbf{0}_{n, \bar{n}} & I_{n, n} & -J I_{n, n} \\
\mathbf{0}_{n, \bar{n}} & \mathbf{0}_{n, n} & I_{n, n}
\end{array}\right) \mid \\
= & \left|\begin{array}{ccc}
J H_{\bar{n}}^{p} & \mathbf{0}_{\bar{n}, n} & \mathbf{0}_{\bar{n}, n} \\
\mathbf{0}_{n, \bar{n}} & H_{n}^{\bar{p}} & \mathbf{0}_{n, n} \\
\left(H_{\bar{n}}^{\bar{p}}\right)_{(\bar{n})} & J H_{n}^{\bar{p}} & -J^{2}\left(H_{n}^{\bar{p}}+H_{n}^{\bar{p}+1}\right)
\end{array}\right| \\
= & (-1)^{n} J \cdot\left|H_{n}^{p+1}\right| \cdot\left|H_{n+1}^{p}\right| \cdot\left|\Sigma_{n}^{p+1}\right| .
\end{aligned}
$$

(L6) Combining (20) and (18), we have

$$
\begin{aligned}
& \left|H_{3 n+2}^{3 p+1}\right|=\left|P^{t} H_{3 n+2}^{3 p+1} P\right| \\
= & \left|\begin{array}{ccc}
J H_{\bar{n}}^{p} & \mathbf{0}_{\bar{n}, \bar{n}} & \left(H_{\bar{n}}^{\bar{p}}\right)^{(\bar{n})} \\
\mathbf{0}_{\overline{\bar{n}}, \bar{n}} & H_{\overline{\bar{p}}}^{\bar{p}} & \left(J H_{\bar{n}}^{\bar{p}}\right)^{(\bar{n})} \\
\left(H_{\bar{n}}^{\bar{p}}\right)_{(\bar{n})} & \left(J H_{\bar{n}}^{\bar{p}}\right)_{(\bar{n})} & \mathbf{0}_{n, n}
\end{array}\right| \\
= & \left.\mid \begin{array}{ccc}
J H_{\bar{n}}^{p} & \mathbf{0}_{\bar{n}, \bar{n}} & \left(H_{\bar{n}}^{\bar{p}}\right)^{(\bar{n})} \\
\mathbf{0}_{\bar{n}, \bar{n}} & H_{\overline{\bar{p}}}^{\bar{p}} & \left(J H_{\bar{n}}^{\bar{p}}\right)^{(\bar{n})} \\
\left(H_{\bar{n}}^{\bar{p}}\right)_{(\bar{n})} & \left(J H_{\bar{n}}^{\bar{p}}\right)_{(\bar{n})} & \mathbf{0}_{n, n}
\end{array}\right)\left(\begin{array}{ccc}
I_{\bar{n}, \bar{n}} & \mathbf{0}_{\bar{n}, \bar{n}} & -J^{2}\left(I_{\bar{n}, \bar{n}}\right)^{(1)} \\
\mathbf{0}_{\bar{n}, \bar{n}} & I_{\bar{n}, \bar{n}} & -J\left(I_{\bar{n}, \bar{n}}\right)^{(\bar{n})} \\
\mathbf{0}_{n, \bar{n}} & \mathbf{0}_{n, \bar{n}} & I_{n, n}
\end{array}\right) \mid
\end{aligned}
$$


$=\left|\begin{array}{ccc}J H_{\bar{n}}^{p} & \mathbf{0}_{\bar{n}, \bar{n}} & \mathbf{0}_{\bar{n}, n} \\ \mathbf{0}_{\overline{\bar{n}}, \bar{n}} & H_{\bar{n}}^{p+1} & \mathbf{0}_{\overline{\bar{n}}, n} \\ \left(H_{\bar{n}}^{\bar{p}}\right)_{(\bar{n})} & \left(J H_{\bar{n}}^{\bar{p}}\right)_{(\bar{n})} & -J^{2}\left(H_{n}^{\bar{p}+1}+H_{n}^{\bar{p}}\right)\end{array}\right|$
$=(-1)^{n} J \cdot\left|H_{n+1}^{p+1}\right| \cdot\left|H_{n+1}^{p}\right| \cdot\left|\Sigma_{n}^{p+1}\right|$.

(L7) Combining (20) and (16), we have

$$
\begin{aligned}
& \left|H_{3 n}^{3 p+2}\right|=\left|P^{t} H_{3 n}^{3 p+2} P\right| \\
& =\left|\begin{array}{ccc}
\mathbf{0}_{n, n} & H_{n}^{\bar{p}} & J H_{n}^{\bar{p}} \\
H_{n}^{\bar{p}} & J H_{n}^{\bar{p}} & \mathbf{0}_{n, n} \\
J H_{n}^{\bar{p}} & \mathbf{0}_{n, n} & H_{n}^{\bar{p}+1}
\end{array}\right| \\
& =\left|\left(\begin{array}{ccc}
\mathbf{0}_{n, n} & H_{n}^{\bar{p}} & J H_{n}^{\bar{p}} \\
H_{n}^{\bar{p}} & J H_{n}^{\bar{p}} & \mathbf{0}_{n, n} \\
J H_{n}^{\bar{p}} & \mathbf{0}_{n, n} & H_{n}^{\bar{p}+1}
\end{array}\right)\left(\begin{array}{ccc}
I_{n, n} & \mathbf{0}_{n, n} & \mathbf{0}_{n, n} \\
-J^{2} I_{n, n} & I_{n, n} & \mathbf{0}_{n, n} \\
J I_{n, n} & -J^{2} I_{n, n} & I_{n, n}
\end{array}\right)\right| \\
& =\left|\begin{array}{ccc}
\mathbf{0}_{n, n} & \mathbf{0}_{n, n} & J H_{n}^{\bar{p}} \\
\mathbf{0}_{n, n} & J H_{n}^{\bar{p}} & \mathbf{0}_{n, n} \\
J\left(H_{n}^{\bar{p}}+H_{n}^{\bar{p}+1}\right) & -J^{2} H_{n}^{\bar{p}+1} & H_{n}^{\bar{p}+1}
\end{array}\right| \\
& =(-1)^{n}\left|H_{n}^{p+1}\right|^{2} \cdot\left|\Sigma_{n}^{p+1}\right| \text {. }
\end{aligned}
$$

(L8) Combining (20) and (17), we have

$$
\begin{aligned}
& \left|H_{3 n+1}^{3 p+2}\right|=\left|P^{t} H_{3 n+1}^{3 p+2} P\right| \\
= & \left|\begin{array}{ccc}
\mathbf{0}_{\bar{n}, \bar{n}} & \left(H_{\bar{n}}^{\bar{p}}\right)^{(\bar{n})} & \left(J H_{\bar{n}}^{\bar{p}}\right)^{(\bar{n})} \\
\left(H_{\bar{n}}^{\bar{p}}\right)_{(\bar{n})} & J H_{n}^{\bar{p}} & \mathbf{0}_{n \times n} \\
\left(J H_{\bar{n}}^{\bar{p}}\right)_{(\bar{n})} & \mathbf{0}_{n \times n} & H_{n}^{\bar{p}+1}
\end{array}\right| \\
= & \left|\begin{array}{ccc}
\mathbf{0}_{\bar{n}, \bar{n}} & \left(H_{\bar{n}}^{\bar{p}}\right)^{(\bar{n})} & \left(J H_{\bar{n}}^{\bar{p}}\right)^{(\bar{n})} \\
\left(H_{\bar{p}}^{\bar{p}}\right)_{(\bar{n})} & J H_{n}^{\bar{p}} & \mathbf{0}_{n \times n} \\
\mathbf{0}_{n, \bar{n}} & -J^{2} H_{n}^{\bar{p}} & H_{n}^{\bar{p}+1}
\end{array}\right| \\
= & 0 .
\end{aligned}
$$

(L9) Combining (20) and (18), we have

$$
\begin{aligned}
& \left|\Sigma_{3 n+2}^{3 p+2}\right|=\left|P^{t} \sum_{3 n+2}^{3 p+2} P\right| \\
& =\left|\begin{array}{ccc}
\mathbf{0}_{\bar{n}, \bar{n}} & H_{\bar{n}}^{\bar{p}} & \left(J H_{\bar{n}}^{\bar{p}}\right)^{(\bar{n})} \\
H_{\overline{\bar{p}}}^{\bar{p}} & J H_{\bar{n}}^{\bar{p}} & \mathbf{0}_{\bar{n}, n} \\
\left(J H_{\bar{n}}^{\bar{p}}\right)_{(\bar{n})} & \mathbf{0}_{n, \bar{n}} & H_{n}^{p+2}
\end{array}\right| \\
& =\left|\left(\begin{array}{ccc}
\mathbf{0}_{\bar{n}, \bar{n}} & H_{\bar{n}}^{\bar{p}} & \left(J H_{\bar{n}}^{\bar{p}}\right)^{(\bar{n})} \\
H_{\overline{\bar{p}}}^{\bar{p}} & J H_{\bar{n}}^{\bar{p}} & \mathbf{0}_{\bar{n}, n} \\
\left(J H_{\bar{n}}^{\bar{p}}\right)_{(\bar{n})} & \mathbf{0}_{n, \bar{n}} & H_{n}^{p+2}
\end{array}\right)\left(\begin{array}{ccc}
I_{\bar{n}, \bar{n}} & -J I_{\bar{n}, \bar{n}} & J^{2} I_{\bar{n}, n} \\
\mathbf{0}_{\bar{n}, \bar{n}} & I_{\bar{n}, \bar{n}} & -J I_{\bar{n}, n} \\
\mathbf{0}_{n, \bar{n}} & \mathbf{0}_{n, \bar{n}} & I_{n, n}
\end{array}\right)\right|
\end{aligned}
$$




$$
\begin{aligned}
& =\left|\begin{array}{ccc}
\mathbf{0}_{\bar{n}, \bar{n}} & H_{\bar{n}}^{\bar{p}} & \mathbf{0}_{\bar{n}, n} \\
H_{\overline{\bar{p}}} & \mathbf{0}_{\bar{n}, \bar{n}} & \mathbf{0}_{\bar{n}, n} \\
\left(J H_{\bar{n}}^{\bar{p}}\right)_{(\bar{n})} & -J^{2}\left(H_{\bar{n}}^{\bar{p}}\right)_{(\bar{n})} & H_{n}^{\bar{p}}+H_{n}^{\bar{p}+1}
\end{array}\right| \\
& =(-1)^{n+1}\left|H_{n+1}^{p+1}\right|^{2} \cdot\left|\Sigma_{n}^{p+1}\right| .
\end{aligned}
$$

\section{Proof of equalities ( L10)-(L18)}

Recall that the sequence $\mathbf{s}=s_{0} s_{1} \cdots s_{n} \cdots \in \mathcal{A}^{\infty}$ is characterized by the recurrence equations in (11), and that $\Sigma_{n}^{p}:=\Sigma_{n}^{p}(\boldsymbol{s})\left(\right.$ resp. $\left.H_{n}^{p}\right)$ is the $(p, n)$-order Hankel matrix of the sequence $\boldsymbol{s}$ (resp. $\boldsymbol{c}$ ). Let $K_{n}^{p}:=$ $K_{n}^{p}(\mathbf{s}):=\left(s_{p+3(i+j-2)}\right)_{1 \leq i, j \leq n}$. By $(11)$, we have for all $n \geq 1, p \geq 0$,

$$
K_{n}^{3 p}=-J^{2} H_{n}^{p}, \quad K_{n}^{3 p+1}=J H_{n}^{p}, \quad K_{n}^{3 p+2}=H_{n}^{p+1} .
$$

Equalities (L10)-(L18) are proved by combining (21) and (16-18) where the sequence $\mathbf{u}$ is specialized to $\mathbf{s}$.

(L10) Combining (21) and (16), we have

$$
\begin{aligned}
& \left|\Sigma_{3 n}^{3 p}\right|=\left|P^{t} \Sigma_{3 n}^{3 p} P\right| \\
& =\left|\begin{array}{ccc}
-J^{2} H_{n}^{p} & J H_{n}^{p} & H_{n}^{\bar{p}} \\
J H_{n}^{p} & H_{n}^{\bar{p}} & -J^{2} H_{n}^{\bar{p}} \\
H_{n}^{\bar{p}} & -J^{2} H_{n}^{\bar{p}} & J H_{n}^{\bar{p}}
\end{array}\right| \\
& =\left|\left(\begin{array}{ccc}
-J^{2} H_{n}^{p} & J H_{n}^{p} & H_{n}^{\bar{p}} \\
J H_{n}^{p} & H_{n}^{\bar{p}} & -J^{2} H_{n}^{\bar{p}} \\
H_{n}^{\bar{p}} & -J^{2} H_{n}^{\bar{p}} & J H_{n}^{\bar{p}}
\end{array}\right)\left(\begin{array}{ccc}
I_{n, n} & J^{2} I_{n, n} & \mathbf{0}_{n, n} \\
\mathbf{0}_{n, n} & I_{n, n} & J^{2} I_{n, n} \\
\mathbf{0}_{n, n} & \mathbf{0}_{n, n} & I_{n, n}
\end{array}\right)\right| \\
& =\left|\begin{array}{ccc}
-J^{2} H_{n}^{p} & \mathbf{0}_{n, n} & H_{n}^{p}+H_{n}^{\bar{p}} \\
J H_{n}^{p} & H_{n}^{p}+H_{n}^{\bar{p}} & \mathbf{0}_{n, n} \\
H_{n}^{\bar{p}} & \mathbf{0}_{n, n} & \mathbf{0}_{n, n}
\end{array}\right| \\
& =(-1)^{n}\left|\Sigma_{n}^{p}\right|^{2} \cdot\left|H_{n}^{p+1}\right| \text {. }
\end{aligned}
$$

(L11) Combining (21) and (17), we have

$$
\begin{aligned}
& \left|\Sigma_{3 n+1}^{3 p}\right|=\left|P^{t} \sum_{3 n+1}^{3 p} P\right| \\
= & \left|\begin{array}{ccc}
-J^{2} H_{\bar{n}}^{p} & \left(J H_{\bar{n}}^{p}\right)^{(\bar{n})} & \left(H_{\bar{p}}^{\bar{p}}\right)^{(\bar{n})} \\
\left(J H_{\bar{n}}^{p}\right)_{(\bar{n})} & H_{n}^{\bar{p}} & -J^{2} H_{n}^{\bar{p}} \\
\left(H_{\bar{n}}^{\bar{p}}\right)_{(\bar{n})} & -J^{2} H_{n}^{\bar{p}} & J H_{n}^{\bar{p}}
\end{array}\right| \\
= & \left|\begin{array}{ccc}
-J^{2} H_{\bar{n}}^{p} & \left(J H_{\bar{n}}^{p}\right)^{(\bar{n})} & \left(H_{\bar{p}}^{\bar{p}}\right)^{(\bar{n})} \\
\left(J H_{\bar{n}}^{p}\right)_{(\bar{n})} & H_{n}^{\bar{p}} & -J^{2} H_{n}^{\bar{p}} \\
\left(H_{\bar{n}}^{\bar{p}}\right)_{(\bar{n})} & -J^{2} H_{n}^{\bar{p}} & J H_{n}^{\bar{p}}
\end{array}\right|
\end{aligned}
$$




$$
\begin{aligned}
& \times\left(\begin{array}{ccc}
I_{\bar{n}, \bar{n}} & J^{2}\left(I_{\bar{n}, \bar{n}}\right)^{(\bar{n})} & J\left(I_{\bar{n}, \bar{n}}\right)^{(1)} \\
\mathbf{0}_{n, \bar{n}} & I_{n, n} & \mathbf{0}_{n, n} \\
\mathbf{0}_{n, \bar{n}} & \mathbf{0}_{n, n} & I_{n, n}
\end{array}\right) \mid \\
& =\left|\begin{array}{ccc}
-J^{2} H_{\bar{n}}^{p} & \mathbf{0}_{\bar{n}, n} & \mathbf{0}_{\bar{n}, n} \\
\left(J H_{\bar{n}}^{p}\right)_{(\bar{n})} & H_{n}^{p}+H_{n}^{\bar{p}} & \mathbf{0}_{n, n} \\
\left(H_{\bar{n}}^{\bar{p}}\right)_{(\bar{n})} & \mathbf{0}_{n, n} & J\left(H_{n}^{\bar{p}}+H_{n}^{\bar{p}+1}\right)
\end{array}\right| \\
& =(-1)^{n+1} J^{2}\left|H_{n+1}^{p}\right| \cdot\left|\Sigma_{n}^{p}\right| \cdot\left|\Sigma_{n}^{p+1}\right| \text {. }
\end{aligned}
$$

(L12) Combining (21) and (18), we have

$$
\begin{aligned}
& \left|\begin{array}{ccc}
\Sigma_{3 n+2}^{3 p}|=| P^{t} \sum_{3 n+2}^{3 p} P \mid \\
= & \left|\begin{array}{ccc}
-J^{2} H_{\bar{n}}^{p} & J H_{\bar{n}}^{p} & \left(H_{\bar{n}}^{\bar{p}}\right)^{(\bar{n})} \\
J H_{\bar{n}}^{p} & H_{\bar{n}}^{\bar{p}} & -J^{2}\left(H_{\bar{n}}^{\bar{p}}\right)^{(\bar{n})} \\
\left(H_{\bar{n}}^{\bar{p}}\right)_{(\bar{n})} & -J^{2}\left(H_{\bar{n}}^{\bar{p}}\right)_{(\bar{n})} & J H_{n}^{\bar{p}}
\end{array}\right| \\
= & \left.\mid \begin{array}{ccc}
-J^{2} H_{\bar{n}}^{p} & J H_{\bar{n}}^{p} & \left(H_{\bar{n}}^{\bar{p}}\right)^{(\bar{n})} \\
J H_{\bar{n}}^{p} & H_{\bar{n}}^{\bar{p}} & -J^{2}\left(H_{\bar{p}}^{\bar{p}}\right)^{(\bar{n})} \\
\left(H_{\bar{n}}^{\bar{p}}\right)_{(\bar{n})} & -J^{2}\left(H_{\bar{n}}^{\bar{p}}\right)_{(\bar{n})} & J H_{n}^{\bar{p}}
\end{array}\right) \\
= & \left.\mid \begin{array}{ccc}
I_{\bar{n}, \bar{n}} & J^{2} I_{\bar{n}, \bar{n}} & J\left(I_{\bar{n}, \bar{n}}\right)^{(1)} \\
\mathbf{0}_{\bar{n}, \bar{n}} & I_{\bar{n}, \bar{n}} & \mathbf{0}_{\bar{n}, n} \\
\mathbf{0}_{n, \bar{n}} & \mathbf{0}_{n, n+1} & I_{n, n}
\end{array}\right) \\
-J^{2} H_{\bar{n}}^{p} & \mathbf{0}_{\bar{n}, \bar{n}} & \mathbf{0}_{\bar{n}, n} \\
J H_{\bar{n}}^{p} & H_{\bar{n}}^{\bar{p}} & \mathbf{0}_{\bar{n}, n} \\
\left(H_{\bar{n}}^{\bar{p}}\right)_{(\bar{n})} & \mathbf{0}_{n, \bar{n}} & J\left(H_{n}^{\bar{p}}+H_{n}^{\bar{p}+1}\right)
\end{array}\right| \\
= & (-1)^{n+1} J^{2}\left|H_{n+1}^{p}\right| \cdot\left|\Sigma_{n+1}^{p}\right| \cdot\left|\Sigma_{n}^{p+1}\right| .
\end{aligned}
$$

(L13) Combining (21) and (16), we have

$$
\begin{aligned}
& \left|\Sigma_{3 n}^{3 p+1}\right|=\left|P^{t} \sum_{3 n}^{3 p+1} P\right| \\
= & \left|\begin{array}{ccc}
J H_{n}^{p} & H_{n}^{\bar{p}} & -J^{2} H_{n}^{\bar{p}} \\
H_{n}^{\bar{p}} & -J^{2} H_{n}^{\bar{p}} & J H_{n}^{\bar{p}} \\
-J^{2} H_{n}^{\bar{p}} & J H_{n}^{\bar{p}} & H_{n}^{\bar{p}+1}
\end{array}\right| \\
= & \left.\mid \begin{array}{ccc}
J H_{n}^{p} & H_{n}^{\bar{p}} & -J^{2} H_{n}^{\bar{p}} \\
H_{n}^{\bar{p}} & -J^{2} H_{n}^{\bar{p}} & J H_{n}^{\bar{p}} \\
-J^{2} H_{n}^{\bar{p}} & J H_{n}^{\bar{p}} & H_{n}^{\bar{p}+1}
\end{array}\right)\left(\begin{array}{ccc}
I_{n, n} & \mathbf{0}_{n \times n} & \mathbf{0}_{n \times n} \\
J I_{n, n} & I_{n, n} & J^{2} I_{n, n} \\
\mathbf{0}_{n \times n} & \mathbf{0}_{n \times n} & I_{n, n}
\end{array}\right) \mid \\
= & \left|\begin{array}{ccc}
J\left(H_{n}^{p}+H_{n}^{\bar{p}}\right) & H_{n}^{\bar{p}} & \mathbf{0}_{n \times n} \\
\mathbf{0}_{n \times n} & -J^{2} H_{n}^{\bar{p}} & \mathbf{0}_{n \times n} \\
\mathbf{0}_{n \times n} & J H_{n}^{\bar{p}} & H_{n}^{\bar{p}}+H_{n}^{\bar{p}+1}
\end{array}\right|
\end{aligned}
$$


$=(-1)^{n}\left|H_{n}^{p+1}\right| \cdot\left|\Sigma_{n}^{p}\right| \cdot\left|\Sigma_{n}^{p+1}\right|$.

(L14) Combining (21) and (17), we have

$$
\begin{aligned}
& \left|\Sigma_{3 n+1}^{3 p+1}\right|=\left|P^{t} \Sigma_{3 n+1}^{3 p+1} P\right| \\
& =\left|\begin{array}{ccc}
J H_{\bar{n}}^{p} & \left(H_{\bar{n}}^{\bar{p}}\right)^{(\bar{n})} & -J^{2}\left(H_{\bar{n}}^{\bar{p}}\right)^{(\bar{n})} \\
\left(H_{\bar{n}}^{\bar{p}}\right)_{(\bar{n})} & -J^{2} H_{n}^{\bar{p}} & J H_{n}^{\bar{p}} \\
-J^{2}\left(H_{\bar{n}}^{\bar{p}}\right)_{(\bar{n})} & J H_{n}^{\bar{p}} & H_{n}^{p+2}
\end{array}\right| \\
& =\mid\left(\begin{array}{ccc}
J H_{\bar{n}}^{p} & \left(H_{\bar{n}}^{\bar{p}}\right)^{(\bar{n})} & -J^{2}\left(H_{\bar{n}}^{\bar{p}}\right)^{(\bar{n})} \\
\left(H_{\bar{n}}^{\bar{p}}\right)_{(\overline{\bar{n}})} & -J^{2} H_{n}^{\bar{p}} & J H_{n}^{\bar{p}} \\
-J^{2}\left(H_{\bar{n}}^{\bar{p}}\right)_{(\bar{n})} & J H_{n}^{\bar{p}} & H_{n}^{p+2}
\end{array}\right) \\
& \times\left(\begin{array}{ccc}
I_{n, n} & -J^{2}\left(I_{\bar{n}, \bar{n}}\right)^{(1)} & J\left(I_{\bar{n}, \bar{n}}\right)^{(1)} \\
\mathbf{0}_{n, \bar{n}} & I_{n, n} & \mathbf{0}_{n, n} \\
\mathbf{0}_{n, \bar{n}} & \mathbf{0}_{n, n} & I_{n, n}
\end{array}\right) \mid \\
& =\left|\begin{array}{ccc}
J H_{\bar{n}}^{p} & \mathbf{0}_{n+1, n} & \mathbf{0}_{\bar{n}, n} \\
\left(H_{\bar{n}}^{\bar{p}}\right)_{(\bar{n})} & -J^{2}\left(H_{n}^{\bar{p}}+H_{n}^{\bar{p}+1}\right) & J\left(H_{n}^{\bar{p}}+H_{n}^{\bar{p}+1}\right) \\
-J^{2}\left(H_{\bar{n}}^{\bar{p}}\right)_{(\bar{n})} & J\left(H_{n}^{\bar{p}}+H_{n}^{\bar{p}+1}\right) & \mathbf{0}_{n, n}
\end{array}\right| \\
& =(-1)^{n} J\left|H_{n+1}^{p}\right| \cdot\left|\Sigma_{n}^{p+1}\right|^{2} \text {. }
\end{aligned}
$$

(L15) Combining (21) and (18), we have

$$
\begin{aligned}
& \left|\Sigma_{3 n+2}^{3 p+1}\right|=\left|P^{t} \Sigma_{3 n+2}^{3 p+1} P\right| \\
& =\left|\begin{array}{ccc}
J H_{\bar{n}}^{p} & H_{\bar{n}}^{\bar{p}} & -J^{2}\left(H_{\bar{n}}^{\bar{p}}\right)^{(\bar{n})} \\
H_{\bar{n}}^{\bar{p}} & -J^{2} H_{\bar{n}}^{\bar{p}} & J\left(H_{\bar{n}}^{\bar{p}}\right)^{(\bar{n})} \\
-J^{2}\left(H_{\bar{n}}^{\bar{p}}\right)_{(\bar{n})} & J\left(H_{\bar{n}}^{\bar{p}}\right)_{(\bar{n})} & H_{n}^{\bar{p}+1}
\end{array}\right| \\
& \left.=\mid \begin{array}{ccc}
J H_{\bar{n}}^{p} & H_{\bar{n}}^{\bar{p}} & -J^{2}\left(H_{\bar{n}}^{\bar{p}}\right)^{(\bar{n})} \\
H_{\bar{n}}^{\bar{p}} & -J^{2} H_{\bar{p}}^{\bar{p}} & J\left(H_{\bar{p}}^{\bar{p}}\right)^{(\bar{n})} \\
-J^{2}\left(H_{\bar{n}}^{\bar{p}}\right)_{(\bar{n})} & J\left(H_{\bar{n}}^{\bar{p}}\right)_{(\bar{n})} & H_{n}^{\bar{p}+1}
\end{array}\right) \\
& \times\left(\begin{array}{ccc}
I_{\bar{n}, \bar{n}} & \mathbf{0}_{\bar{n}, \bar{n}} & \mathbf{0}_{\bar{n}, n} \\
J I_{\bar{n}, \bar{n}} & I_{\bar{n}, \bar{n}} & J^{2}\left(I_{\bar{n}, \bar{n}}\right)^{(\bar{n})} \\
\mathbf{0}_{n, \bar{n}} & \mathbf{0}_{n, \bar{n}} & I_{n, n}
\end{array}\right) \\
& =\left|\begin{array}{ccc}
J\left(H_{\bar{n}}^{p}+H_{\bar{n}}^{\bar{p}}\right) & H_{\bar{n}}^{\bar{p}} & \mathbf{0}_{\bar{n}, n} \\
\mathbf{0}_{\bar{n}, \bar{n}} & -J^{2} H_{\bar{n}}^{\bar{p}} & \mathbf{0}_{\bar{n}, n} \\
\mathbf{0}_{n, \bar{n}} & J\left(H_{\bar{n}}^{\bar{p}}\right)_{(\bar{n})} & H_{n}^{\bar{p}}+H_{n}^{\bar{p}+1}
\end{array}\right| \\
& =(-1)^{n+1}\left|H_{n+1}^{p+1}\right| \cdot\left|\Sigma_{n+1}^{p}\right| \cdot\left|\Sigma_{n}^{p+1}\right| \text {. }
\end{aligned}
$$


(L16) Combining (21) and (16), we have

$$
\begin{aligned}
& \left|\Sigma_{3 n}^{3 p+2}\right|=\left|P^{t} \Sigma_{3 n}^{3 p+2} P\right| \\
= & \left|\begin{array}{ccc}
H_{n}^{\bar{p}} & -J^{2} H_{n}^{\bar{p}} & J H_{n}^{\bar{p}} \\
-J^{2} H_{n}^{\bar{p}} & J H_{n}^{\bar{p}} & H_{n}^{\bar{p}+1} \\
J H_{n}^{\bar{p}} & H_{n}^{\bar{p}+1} & -J^{2} H_{n}^{\bar{p}+1}
\end{array}\right| \\
= & \left.\mid \begin{array}{ccc}
H_{n}^{\bar{p}} & -J^{2} H_{n}^{\bar{p}} & J H_{n}^{\bar{p}} \\
-J^{2} H_{n}^{\bar{p}} & J H_{n}^{\bar{p}} & H_{n}^{\bar{p}+1} \\
J H_{n}^{\bar{p}} & H_{n}^{\bar{p}+1} & -J^{2} H_{n}^{\bar{p}+1}
\end{array}\right)\left(\begin{array}{ccc}
I_{n, n} & \mathbf{0}_{n, n} & -J I_{n, n} \\
\mathbf{0}_{n, n} & I_{n, n} & \mathbf{0}_{n, n} \\
\mathbf{0}_{n, n} & J I_{n, n} & I_{n, n}
\end{array}\right) \mid \\
= & \left|\begin{array}{ccc}
H_{n}^{\bar{p}} & \mathbf{0}_{n, n} & \mathbf{0}_{n, n} \\
-J^{2} H_{n}^{\bar{p}} & J H_{n}^{\bar{p}} & H_{n}^{\bar{p}}+H_{n}^{\bar{p}+1} \\
J H_{n}^{\bar{p}} & \mathbf{0}_{n, n} & -J^{2}\left(H_{n}^{\bar{p}}+H_{n}^{\bar{p}+1}\right)
\end{array}\right| \\
= & (-1)^{n}\left|\Sigma_{n}^{p+1}\right|^{2} \cdot\left|H_{n}^{p+1}\right| .
\end{aligned}
$$

(L17) Combining (21) and (17), we have

$$
\begin{aligned}
& \left|\Sigma_{3 n+1}^{3 p+2}\right|=\left|P^{t} \Sigma_{3 n+1}^{3 p+2} P\right| \\
& =\left|\begin{array}{crc}
H_{\bar{n}}^{\bar{p}} & -J^{2}\left(H_{\bar{p}}^{\bar{p}}\right)^{(\bar{n})} & J\left(H_{\bar{n}}^{\bar{p}}\right)^{(\bar{n})} \\
-J^{2}\left(H_{\bar{n}}^{\bar{p}}\right)_{(\bar{n})} & J H_{n}^{\bar{p}} & H_{n}^{\bar{p}+1} \\
J\left(H_{\bar{n}}^{\bar{p}}\right)_{(\bar{n})} & H_{n}^{\bar{p}+1} & -J^{2} H_{n}^{\bar{p}+1}
\end{array}\right| \\
& \left.=\mid \begin{array}{ccc}
H_{\bar{n}}^{\bar{p}} & -J^{2}\left(H_{\bar{n}}^{\bar{p}}\right)^{(\bar{n})} & J\left(H_{\bar{n}}^{\bar{p}}\right)^{(\bar{n})} \\
-J^{2}\left(H_{\bar{n}}^{\bar{p}}\right)_{(\bar{n})} & J H_{n}^{\bar{p}} & H_{n}^{\bar{p}+1} \\
J\left(H_{\bar{n}}^{\bar{p}}\right)_{(\bar{n})} & H_{n}^{\bar{p}+1} & -J^{2} H_{n}^{\bar{p}+1}
\end{array}\right) \\
& \times\left(\begin{array}{ccc}
I_{\bar{n}, \bar{n}} & \mathbf{0}_{\bar{n}, n} & -J\left(I_{\bar{n}, \bar{n}}\right)^{(\bar{n})} \\
\mathbf{0}_{n, \bar{n}} & I_{n, n} & \mathbf{0}_{n, n} \\
\mathbf{0}_{n, \bar{n}} & J I_{n, n} & I_{n, n}
\end{array}\right) \mid \\
& =\left|\begin{array}{ccc}
H_{\bar{n}}^{\bar{p}} & \mathbf{0}_{\bar{n}, n} & \mathbf{0}_{\bar{n}, n} \\
-J^{2}\left(H_{\bar{n}}^{\bar{p}}\right)_{(\bar{n})} & J\left(H_{n}^{\bar{p}}+H_{n}^{\bar{p}+1}\right) & H_{n}^{\bar{p}}+H_{n}^{\bar{p}+1} \\
J\left(H_{\bar{n}}^{\bar{p}}\right)_{(\bar{n})} & \mathbf{0}_{n, n} & -J^{2}\left(H_{n}^{\bar{p}}+H_{n}^{\bar{p}+1}\right)
\end{array}\right| \\
& =(-1)^{n}\left|\sum_{n}^{p+1}\right|^{2} \cdot\left|H_{n+1}^{p+1}\right| \text {. }
\end{aligned}
$$

(L18) Combining (21) and (18), we have

$$
\begin{aligned}
& \left|\Sigma_{3 n+2}^{3 p+2}\right|=\left|P^{t} \sum_{3 n+2}^{3 p+2} P\right| \\
& =\left|\begin{array}{ccc}
H_{\bar{n}}^{\bar{p}} & -J^{2} H_{\bar{n}}^{\bar{p}} & J\left(H_{\bar{n}}^{\bar{p}}\right)^{(\bar{n})} \\
-J^{2} H_{\bar{n}}^{\bar{p}} & J H_{\bar{n}}^{\bar{p}} & \left(H_{\bar{n}}^{\bar{p}+1}\right)^{(\bar{n})} \\
J\left(H_{\bar{n}}^{\bar{p}}\right)_{(\bar{n})} & \left(H_{\bar{n}}^{\bar{p}+1}\right)_{(\bar{n})} & -J^{2} H_{n}^{\bar{p}+1}
\end{array}\right|
\end{aligned}
$$


$=\left|\begin{array}{ccc}H_{\bar{n}}^{\bar{p}} & \mathbf{0}_{\bar{n}, \bar{n}} & J\left(H_{\bar{p}}^{\bar{p}}\right)^{(\bar{n})} \\ -J^{2} H_{\bar{n}}^{\bar{p}} & \mathbf{0}_{\overline{\bar{n}}, \bar{n}} & \left(H_{\bar{n}}^{\bar{p}+1}\right)^{(\bar{n})} \\ J\left(H_{\bar{n}}^{\bar{p}}\right)_{(\bar{n})} & \left(\Sigma_{\bar{n}}^{\bar{p}}\right)_{(\bar{n})} & -J^{2} H_{n}^{\bar{p}+1}\end{array}\right|$
$=0$.

\section{CONCluding Remarks}

It is worth mentioning that we do not have a unified result, even though Bacher's result and ours are very similar. Let $H_{n}^{A}, H_{n}^{B}, H_{n}^{C}, H_{n}^{D}$ be the Hankel determinants of the Thue-Morse-like sequence defined by the following generating functions respectively:

$$
\begin{gathered}
P^{A}(x)=\prod_{k \geq 0}\left(1-x^{2^{k}}\right), \\
P^{B}(x)=\prod_{k \geq 0}\left(1+J x^{3^{k}}\right), \\
P^{C}(x)=\prod_{k \geq 0}\left(1+Y x^{5^{k}}\right), \\
P^{D}(x)=\prod_{k \geq 0}\left(1+I x^{2^{k}}\right),
\end{gathered}
$$

where $Y=e^{2 i \pi / 5}$. The first values of these Hankel determinants are reproduced in Table 1.

As shown by Bacher and the authors, the determinants $H_{n}^{D}$ and $H_{n}^{B}$ have simple expressions. Unfortunately, the determinants $H_{n}^{A}$ and $H_{n}^{C}$ do not seem to have any simple expressions. Hence, we do not have a unified theory about the Hankel determinants of the sequence defined by the generating function of the form $\prod_{k \geq 0}\left(1+\xi x^{d^{k}}\right)$.

\section{REFERENCES}

[1] J. P. Allouche, J. Peyrière, Z.-X. Wen and Z.-Y. Wen, Hankel determinants of the Thue-Morse sequence, Ann. Inst. Fourier, Grenoble, 48, 1 (1998), 1-27.

[2] J. P. Allouche and J. Shallit, Automatic sequences: Theory, Applications, Generalizations, Cambridge University Press 2003.

[3] R. Bacher, La suite de Thue-Morse et la catégorie Rec, C. R. Acad. Sci. Paris, Ser. I, 342, (2006), 161-164

[4] R. Bacher, Recurrence matrices, arxiv.org/abs/math/0601372, (2006), 80 pages

[5] G. A. Jr. Baker and P. Graves-Morris, Padé approximations, second edition. Cambridge University Press, 1996. 


\begin{tabular}{|c|c|c|c|c|}
\hline$n$ & $H_{n}^{A}$ & $H_{n}^{D}$ & $H_{n}^{C}$ & $H_{n}^{D}$ \\
\hline 0 & 1 & 1 & 1 & 1 \\
\hline 1 & 1 & 1 & 1 & 1 \\
\hline 2 & -2 & $J+1$ & $-Y^{2}$ & $I+1$ \\
\hline 3 & 4 & 1 & 0 & $2 I$ \\
\hline 4 & 8 & $J+1$ & $-Y^{3}-Y^{2}-Y-1$ & $2 I+2$ \\
\hline 5 & -16 & $J$ & $-Y^{2}-Y-1$ & $4 I$ \\
\hline 6 & -32 & $J+1$ & $-Y-1$ & $4 I-4$ \\
\hline 7 & -64 & 1 & $Y^{3}+Y^{2}+Y+1$ & $8 I$ \\
\hline 8 & 128 & $J+1$ & 0 & $8 I+8$ \\
\hline 9 & -256 & 1 & 0 & $16 I$ \\
\hline 10 & -1536 & $J+1$ & 0 & $16 I-16$ \\
\hline 11 & -3072 & $J$ & 0 & -32 \\
\hline 12 & 2048 & $J+1$ & 0 & $32 I-32$ \\
\hline 13 & 4096 & $J$ & 0 & $64 I$ \\
\hline 14 & 8192 & -1 & 0 & $64 I-64$ \\
\hline 15 & -16384 & $J$ & 0 & $128 I$ \\
\hline 16 & 32768 & $J+1$ & 0 & $128 I+128$ \\
\hline 17 & -65536 & $J$ & 0 & $256 I$ \\
\hline 18 & -393216 & $J+1$ & 0 & $256 I-256$ \\
\hline 19 & -2359296 & 1 & $-Y$ & -512 \\
\hline 20 & 14155776 & $J+1$ & $-Y^{3}-2 Y^{2}-2 Y-1$ & $512 I-512$ \\
\hline 21 & 28311552 & 1 & $-2 Y^{2}-3 Y-2$ & -1024 \\
\hline 22 & -94371840 & $J+1$ & $Y^{3}-2 Y-2$ & $-1024 I-1024$ \\
\hline 23 & 62914560 & $J$ & 0 & -2048 \\
\hline 24 & 8388608 & $J+1$ & $3 Y^{3}+8 Y^{2}+8 Y+3$ & $2048 I-2048$ \\
\hline 25 & 16777216 & 1 & $8 Y^{2}+13 Y+8$ & $4096 I$ \\
\hline
\end{tabular}

TABLE 1. The Hankel determinants $H_{n}^{A}, H_{n}^{B}, H_{n}^{C}, H_{n}^{D}$

[6] C. Brezinski, Padé type Approximation and General Orthogonal Polynomials, International Series of Numerical Mathematics. Vol. 50, Birkhäuser Verlag, 1980.

[7] Y. Bugeaud, On the rational approximation to the Thue-Morse-Mahler numbers, Ann. Inst. Fourier, 61 no. 5 (2011),2065-2076.

[8] Y.-J. Guo, Z.-X. Wen and W. Wu, On the irrationality exponent of the regular paperfolding numbers, Linear Algebra Appl. 446 (2014), 237-264.

[9] G.-N. Han, Hankel determinant calculus for the Thue-Morse and related sequences, J. Number Theory, to appear, 2014, 22 pages.

[10] G.-N. Han, Hankel continued fraction and its applications, submitted, (2014), 22 pages

[11] T. Kamae, J. Tamura and Z.-Y. Wen, Hankel determinants for the Fibonacci word and Padé approximation, Acta Arith. 89 (1999) 123-161. 
[12] J. A. Shohat and J. D. Tamarkin, The Problem of Moments, New York: American Mathematical Society (1943).

[13] J. Tamura, Padé approximation for the infinite words generated by certain substitutions, and Hankel determinants, in: Number Theory and Its Applications, K. Győry and S. Kanemitsu (eds.), Kluwer Academic Publishers. (1999), 309-346.

(G.-N. Han) Institut de Recherche Mathématique Avancée, Université de Strasbourg et CNRS, 7 Rue René Descartes, 67084 Strasbourg, FRANCE

E-mail address: guoniu.han@unistra.fr

(W. Wu) Department of Mathematics, Hubei University, 430062, Wuhan, P. R. China

Department of Mathematics Science, P. O. Box 3000, 90014 UniverSITY OF OULU, Finland

E-mail address, Corresponding author: hust.wuwen@gmail.com 